\title{
A Misfit Tool: Patients' (Lack of) Use of a Structured Decision Aid
}

\author{
Jenna L. Marquard \\ College of Engineering \\ University of Massachusetts, Amherst \\ marquard@engin.umass.edu
}

\author{
Patricia Flatley Brennan \\ College of Engineering \\ University of Wisconsin - Madison \\ pbrennan@ie.engr.wisc.edu
}

\begin{abstract}
Motivation - Patients face a new and complex set of choices in determining whether to electronically share their medication information with care providers. Research approach - We designed and tested a scorecard-type decision aid that structured and presented the benefits and concerns of sharing medication information to patients. Findings/Design - Patients' sharing choices and level of understanding did not vary based on their access to the decision aid, and they often did not use the aid. Research limitations/Implications - We tested the decision aid with only neurology patients, limiting the generalizability of the findings. Originality/Value - Our study describes an instance where decisionmakers without high levels of understanding felt confident about their ability to make choices and therefore did not reference a readily available decision aid. Take away message - Decision aids, to be more useful and widely used, should be designed based on decision-makers' behavioral manner of decision-making instead of normative decision-making paradigms.
\end{abstract}

\section{Keywords}

Health care, informatics, decision aid, decision-making

\section{INTRODUCTION}

As health information technologies such as personal health records (PHRs) - a lifelong copy of an individual's health information - become more prevalent, patients will face a new and complex set of choices in determining whether to electronically share their medication information with care providers. Both policy groups and those designing technical platforms for PHRs describe a unified patient medication-list as an early application of PHRs (Tang et al., 2006).

Patients' choices to electronically share medication information can be grouped under decisions named choices under uncertainty, which occur when decision-makers must choose among a set of alternatives, and when the outcomes associated with those alternatives are uncertain. Lay decision-makers will need to choose among two alternatives for sharing information about each of their medications - share or do not share - with those alternatives each having various attributes and potential outcomes. Patients may realize clinical care benefits from sharing, including fewer medication interactions and improved follow-up care and monitoring (Hadjikoutis, Morgan, Wild, \& Smith, 2005). Patients may also see information-disclosure concerns realized from sharing, such as social labeling by their care physician and use of their information for reasons unrelated to their care (Lelloitt, 2003). While the numerical likelihoods of these outcomes are unknown, relative levels of likelihood can be estimated based on known information about an individual's medications.

While surveys show that patients are concerned about the privacy, security and perverse use of their health information, decision-making heuristics and biases may affect patients' choices to electronically share their health information (Markle Foundation, 2005). For instance, the availability heuristic may cause patients to overestimate the occurrence of either clinical care benefits or information disclosure concerns, thus influencing their sharing choice(s). When decisions are complex and uncertain, as is the case here, systematic analyses can allow for more comprehensive structuring of the situation, organizing information with which unaided decision-makers may be unable to cope (Greenberger, Crenson \& Crissey, 1976). Patients, it seems, would be ideal candidates for a simple decision aid that could structure and present these uncertain benefits and concerns to them before they make their sharing choices.

\section{DESIGN OF THE DECISION AID}

We aimed to supplement existing decision-maker knowledge and experience with a structured decision aid, guided by the characteristics of choices under uncertainty - making these characteristics visible and understandable to participants. The decision aid was not aimed at providing participants with suggested choices, but at providing information that the decision-maker likely would not generate and/or structure on his/her own and could use to inform his/her choices.

We designed the decision aid using a scorecard format, which has known benefits of understandability, minimal overlay of analysts' values and biases and an ability to retain multidimensionality (Miser \& Quade, 1985). Along the top of the 
scorecard were headers for four columns, two addressed potential clinical care benefits related to sharing their medication information: reduced potential for interactions and aiding follow-up care and monitoring and two addressed potential information-disclosure concerns related to sharing their medication information: increased potential for social labeling and increased potential use of the information other than for your [the patient's] direct care. Each scorecard cell corresponded to the rating for a particular medication - low, moderate and high - on one of the four categories. These ratings were displayed as either written words or colored circles - red for high, yellow for moderate and green for low. The ratings were judged by a Registered Nurse / Nurse-Practitioner.

\section{METHOD}

To understand how the decision aid impacted decision-makers' understanding and choices, 31 study participants were shown either no decision aid or one of the two versions of the decision aid. All participants were recruited from a neurology clinic in Madison, WI, USA. The presentation of no decision aid served as a baseline measure of the sharing choices patients would make independent of any decision aid - the likely scenario. While viewing the decision aid, participants completed a realistic decision scenario where they chose whether to share their medication information with three types of physicians. We measured participants' sharing choices, their reason(s) for making their choices, and their understanding of the potential consequences of their choices (Kaser-Boyd, Adelman, Taylor \& Nelson, 1986).

\section{RESULTS}

Participants' choices did not vary based on whether they viewed any decision aid or what type of decision aid they viewed. Additionally, participants who viewed one of the decision aids did not more clearly understand the potential implications of their choices. In fact, participants largely appeared not to use the decision aid in their decision making. In this case, analytical information did not supplement decision-maker knowledge and experience as expected, even when participants' level of understanding was not high. A subset of participants acknowledged the decision aid but dismissed its usefulness. One participant commented that the choices were "pretty clear even without that information" while another stated the decision aid was "just another person's opinion." In general, participants did not view their choices in the structured manner proposed by the decision aid. Instead, past experiences, fear of medication interactions and trust in their physicians drove their choices.

\section{DISCUSSION}

This work was premised on the idea that decision-makers would use the decision aid in their decision making. Yet, study participants did not appear to use the decision aid as they made their choices. There are important implications of these null study findings. These findings are important because they describe an instance where decision-makers without high levels of understanding felt confident about their ability to make choices and did not reference a readily available decision aid as they made their choices.

We did not assess patients' behavioral-style of decision-making prior to creating the decision aid - instead we designed the decision aid based on a normative model of decision-making. While we regret not accounting for patients' decisionmaking strategies, decision aids based on normative decision-making approaches are commonplace - and thus are often discarded. Decision aids, to be more useful and widely used, should be designed based on decision-makers' behavioral manner of decision-making instead of normative decision-making paradigms.

\section{ACKNOWLEDGMENTS}

We thank the members of the Health Systems Lab for their support. We also thank Amy Brady and the conference reviewers for their thoughtful suggestions.

\section{REFERENCES}

Greenberger, M., Crenson, M. A. \& Crissey, B. L. (1976) Models in the policy process: Public decision making in the computer era, New York, Russell Sage Foundation.

Kaser-Boyd, N., Adelman, H. S., Taylor, L. \& Nelson, P. (1986) Children's understanding of risks and benefits of psychotherapy, Journal of Clinical Child Psychology, 15, 2, 165.

Lelliott, P. (2003). Secondary uses of patient information, Advances in Psychiatric Treatment, 9, 3, 221-228.

Markle Foundation. (2005) Americans support online personal health records; patient privacy and control over their own information are crucial to acceptance, Retrieved October 2, 2007, from http://www.markle.org/resources/press_center/press_releases/2005/press_release_10112005.php

Michels, R. D. \& Meisel, S. B. (2003) Program using pharmacy technicians to obtain medication histories, American Journal of Health-System Pharmacy, 60, 19, 1982-1986.

Miser, H. \& Quade, E. (Eds.). (1985) Handbook of systems analysis: Overview of uses, procedures, applications, and practice, New York, Elsevier.

Tang, P. C., Ash, J. S., Bates, D. W., Overhage, J. M. \& Sands, D. Z. (2006) Personal health records: Definitions, benefits, and strategies for overcoming barriers to adoption, Journal of the American Medical Informatics Association, 13, 2, 121. 